\section{CONTRIBUIC̣ĀO AO ESTUDO DOS ELEMENTOS VASCULARES, ARTERIAIS E VENOSOS, DO HILO RENAL, EM BOVINOS DA RACCA NELORE}

GRANI NAPICI EOMFIM MARIANA Professcra Hssistente Doutora Faculdade de Medicina Veterinaria e Zootecnia da USP

UICENTE EORELLI Professor Titular caculade de Madicina Veterinśria e Zootecnia da USP
INTROEUCAD

Considerando-se que os rins sõo orincipeis orgãos de filtracäo zangulnea do organismo animal, è natural que estes venham sendo objeto de eztudo de varios pesquisadores, tanto no campo macroscopico, como no uliraestrutura!. Entrebanto, notamos que são poucos e imorecisos os conhecimentos atinentes aos vasos hilares dos rins dos bovinos. Lembrando que, particularmente os zebuinos, erroora constituindo-se hoje, em animais de valor economico incontestavel, vam sendo pouco explorados cintificamente, procuramos neste trabalho, investigar a regiăo hilar dos bovinos de origem indiana da raca ivelore, a im de identificar o numero e a oisposicaro dos ramos arteriais e raizes venosas das arterias e veias renais, contribuindo, assim, para - conhecimento mais pormenorizado destas estruturas.

MATERIAL E METODO

Para a realizaço desía trabalho utilizamos 30 pares de rins de bovinos da raça Nelore, 15 fêmeas (Obs 1 a 15) e 15 machos (Obs 16 a 30 ), acultos, provenientes do Municlpio de Itapolis - SP.

Logo apos o sacrifleio dos animais, retiramos os orgaros as pares, com suas respectivas arterias e veias, unidas a artéria aorta e veia cava caudal. A seguir, íxamos este material injetando solucao aquosa de formol a $10 \%$ nas artérias reneis, conservando posteriprmente as pecas inversas em igual solufaro $e$ depois, de no minimo 48 horas, mediante dissecfăo, individualizamos as componentes do pediculo renal, executando antes a contratorsao do rim esqueido, no sentido mediolateral, manteno para tanto fixada a sua porço cranial.

Para a descricao dos resultados demarcamos no hilo renal os quadrantes craniolateral, craniomedial, caudolateral e cauromedial, a custa de duas linhas, uma long:-idinal ce polo a polo do rim, representando o Jiàmetro craniocaudal e outra, correspondente ao diametro lateraiateral, nargendicular d primeirs, interceptando-a no seu cantro geornetrico, sendo que consideränos ccufando posicáo central ou periferica, os vasos encontredos respectivamente, na metade geometrica contral ou gerifarica
UNITERMOS: Dnatomia, bovinos; Rim; Arterias; Veias 
do hilo rena!, que apresenta farma eipsúide.

Realizamos, ainda, desenthos esquemáticas re todas as prejaracoes (Figs. 1 a 30), ذeri como fotos pare a devida documentäça (Figs. 31 e $32 j$ e, anábise estatlstice dos resultados, onde aplicamos o teste $x:(\omega=5 \%)$

\section{RESULTAROS}

Rs observacơes correspondentes ao numero a disposiczo dos ramos arteriais e das ralzes venasas, pealizadas nos 30 pares de rins de tovinos da raca Nelore, segundo as critérios estabelecidos, possibilitaram os resultados expostos nos quadros $1,2,3,4$.

\section{COMENTARIOS}

Consioeranjo, inicialmente, o ndmero de ramos arteriáis encontrados na regiao hilar, cevemos iemorar que, nos bovinos da raca Nelore, estes ocorrem no rim.direito, em numero de trés - 5 vezes $(.16,6 \% \pm 6,8)$, quatro - 11 vezes. $(36,6 \%$ $\pm 8,8)$, cinco- 3 vezes $(10,0 \% \pm 5,5)$, seis -7 vezes $(23,3 \%+7,7)$, sete -3 vezes $(10,0 \% \pm 5,5)$, oito -1 vez $(3,3 \%$ $\pm 3,3)$ e no rim esquerdo, três - 6 vezes $(20,0 \% \pm 7,3)$, quatro- 91 vezes $(36,6 \%$ $\pm 8,8)$, cinco- 4 vezes $(13,3 \% \pm 6,2)$, seis - 6 vezes $(20,0 \% \pm 7,3)$, sete -2 vezes $(6,6 \% \pm 4,5)$ e nove - 1 vez $(3,3 \%$ $+3,3)$. Estes resultados mostram variaça de três a nove artésias na regiao hilar, com maior freooência de quatro, e uma mediana tanto para o rim direito como para o esquerdo de 5,5. Confrontando estes dados com os da literatura. podemos de inicio observar que estes ngo coinciden totalmente com os apresentados pelos tratadistas, que informam a exis tência de dois a três ramos cBOSSI et alii, 1; ZIMMERL et alii, 21; FAUILLI, 5) ou cinco a oito ramos (LEISERING \& MUELLER， 9; ELLENBERGER \& BAUM，4; BRUNI \& ZIMMERL, 2; SISSDN \& GROSSMAN, 19; SCHWARZE \& SCHRODER, 18) e nao podem ser cotejados com aqueles que simplesmente mencionam a presenca de inumeros vasos GMOHGIARDINO, 15; MARTIN, 12; LEPOUTRE, $10 ;$ LESBRE,. 11; DOBBERSTEIN a HOFFMANN, 3i KOCH, Q; NICKEL et a ¿̇i, 17; GETTY,
6) que ngo fazem especificamente olus\%o ao numero de ramos (MASSUI, 14; GOAZZHLEZ y GAREIA \& GCNIRLEZ ALVAREZ, T) OU QUe genericamenta referem-se a numerosos Vasos (MONTANE \& BQURDELIE, TE) OU a Inca, com os que simplesmente esciarecem que a arteria renal bifurca-se ao alcancar a regiao hilar (MARTIN \& SHAUDER, i3). Jé, nos taurinos da raca Hereford (TEIXEIRA FILHO, 20), as arterías na regiao hilar variam de trés a quinze, com maior frecoência de seis e uma mediana de 7,5 para ambos os rins, valores que se acham muito acima dos encontrados nos zebulinos ora estudados, caracteri$z$ ando provavelmente mais una diferenca racial entre estes animais. Relativamente às ralzes venosas, identificamos na regigo hilar dos bovinos da raca Nelore, no $r$ im direito em numero de duas - 1 vez $(3,3 \% \pm 3,3)$, três - 3 vezes $(10,0 \% \pm$ $5,5)$, quatro - 5 vezes $(16,6 \% \pm 6,8)$, cinco - 9 vezes $(30,0 \% \pm 8,4)$, seis - 4 vezes $(13,3 \% \pm 6,2)$, sete -3 vezes $(10,0 \%+5,5)$, dez-1 vez $(3,3 \% \pm 3,3)$ e no rim esquerdo, três - 2 vezes $(6,8$ : $+4,5)$, quatro- 5 vezes $(16,6 \% \pm 6,8)$, cinco- 5 vezes $(16,6 \% \pm 6,8)$, seis -7 vezes $(23,3 \% \pm 7,7)$, sete -4 vezes $(13,3 \% \pm 6,2)$, oito- 5 vezes $(16,6 \%$ 6,8), nove - 1 vez $(3,3 \% \pm 3,3)$ e dez 1 vez $(3,3 \% \pm 3,3)$, revelam cue estes vasos apresentam variacgo de duas a dez ralzes, com maior frequencia de cinco e uma mediana de 6,0 para o rim direito e 6,5 para o esquerdo. Estes achados, apresentam-se discordantes em relaço a maioria dos tratadistas que apenas referem uma veia renal (MARTIN, 12; LEPOUTRE, 10; LESBRE, 11; ZIMMERL et ali 21; ELLENBERGER \& BAUM, 4 ; BRUNI \& ZIMMERL, 2; MASSUI, 14; DOBPERSTEIN \& HOFFMAN, 3 ; SCHWARZE \& SCHRODER, 18 ; NICKEL et alii, 17) fato nunca identificado em nosso material e ainda impossivel de ser confiontado com aqueles que apenas indicam que esta veia resulta ca uniro de inumeros vasos (LEISERING \& MUELLER, 9) e muito semelhante $\dot{a}$ informacgo dos autores, que regietram a presenca de quatro a cinco ralzes venosas (BDSSI et alii, 1). De outra parte, nos bovinos de origem européia da raca Hereford (TEIXEIRA FILHO, 20), as veias sao vistas apresentando de trés a treze ra!zes, com maior freqoência de oite a uma mediana de 7,5 para o rim direito e 7,0 para o esquerdo, o que também muito difere dos resultados agora identificados para os animais da rata lieloro.

Quanto ao povoamento dos quadrantes felos ramos arteriais, cabe destaque o rato de que, tanto nos bovinus ó raca Hereford, como nos da raca Nelure, en 
ambes os rins, o quadrante cranionedial apresenta maior numero oe arterias, seguico pelos quadrantes caucomedial, creniolateral e caudolateral, apeser ia diference numerica dos ramos arteriajs encontrados na regico hibar destes dois grupos de animais. No atinente as ralzes venosas, observancs que nestas duas racas, o quadrante caudolateral apresenta-se tanto no rim direito, como no esquerdo, como o menos povoado e tambem com reląăo às orterias, abrigando quase sempre nestes zebulncs o ureter, sendo que o maior numero de veias ocorre, por ordem, nos quadrante craniolateral, caudomedial e craniomedial, nos bovinos da raca Hereford em ambos os ríns e nos Nelore, nos quadrantes craniomedial, craniolateral e caudomedial no rim direitc e craniolateral, craniomedial e caudomedial, no rim esquerdo, existindo portanto, apenas coincidencia parcial, no que tange a disposicgo das ralzes venosas nestes especimes.

Rinda, igual numero.de ramos arteriais, bem como de ralzes venosas, ocorrem ocasionalmente em ambos os rins de um mesmo animal, tanto nos bovinos da raca Nelore ( 9 e 6 vezes) como nos da raça Hereford ( 6 e 8 vezes).

De outra parte, considerando o aspecto global dos ramos arterieis, bem como das ralzes venosas, pudemos noter que estes vasos, tanto nos bovinos de raca Nelore, como nos Hereford, ocupam mais freqoentemente, por ordem, oosicoes predominantemente periférica, exclusivamente periferica e predominantemente central, sendo que algumas diferencas podem ser identificadas, pois, nestes zebulnos, identificamos estes vasos em alguns casos em posicão exclusivamente central ou igualmente central e periferica, fato que entendemos deva ter ocorrico em funcro do criterio aootádo para o registro destas variacbes.

Nesta analise realizada entre os dados obtidos para bovinos das racas Nelore e Hereford, sobressai o fato de que, nestes zebuinos, ngo existem diferencas atinentes ao sexo, quando consideramos o numero de ramos arteriais e de ralzes venosas, tanto no rim direito, como no esquerdo, enquanto nos taurinos da raca Hereford, este acontecimento so ocorre em relacgo as ralzes venosas à esquerda.

Devemos, finalmente, salientar que procuramos destacar as principais diferencas encontradas entre os taurinos e zebuinos, isto e, entre os bovinos de racas hereford (TEIXEIRA FILHO, 20) e Nelore (ora estudados), a fim de identi- ficar caracteristicas provevelmente raciais de incontestavel importancia para - melhor reconhecimento destes animais e funcamentais para o desenvolvimento da Anatomá Comparade.

\section{C๐Nட:USSES}

Os resultados de estudos ora realizados, sobre os ramos arteriais e ralzes venosas, dos 30 pares de rins de bovinos da raca Nelore, adultos, 15 machos e 15 fêmeas, permitiram as seguintes conclusơes:

1) os ramos arteriais da regigo hilar aparecem no rim direito, em ndmero de quatro $(36,6 \%)$, seis $(23,3 \%)$, três $(16,6 \%)$, sete $(10,0 \%)$, cinco $(10,0 \%)$, oito $(3,3 \%)$ e no rim esquerdo, en nemero de quatro $(36,6 \%)$, seis $(20,0 \%)$, três $(20,0 \%)$, cinco $(13,3 \%)$ sete $(6,6 \%)$, nove $(3,3 \%)$;

2) as ralzes venosas nessa regizo, surgem no rim dirajto, em numero de cinco $(30,0 \%)$, quatro $(16,6 \%)$, seis $(13,3 \%)$, nove $(10,0 \%)$, sete $(10,0 \%)$, oito $(6,6 \%)$, três $(6,6 \%)$, dez $(3,3 \%)$, dois $(3,3 \%)$, e, no rim esquerdo, en numero de seis $(23,3 \%)$, oito $(16,6 \%)$, cinco $(16,6 \%)$, quatro $(16,6 \%)$, sete $(13,3 \%)$, trés $(6,6 \%)$, dez $(3,3 \%)$, nove $(3,3 \%)$;

3) tanto no rim direito como no esquerdo, o quadrante craniomedial e - mais ojensamente povoado pelos ramos arteriajs, seguido dos qua. crantes cauoumedial, craniclateral e cauciolateral;

4) no rim direltc, no quadrante craniomedial, encontra-se maior numero de ralzes venosas, seguido pelos quadrantes craniolateral e igualmente caudomedial e caudolateral, enquanto que no rim esquerdo estes vasos sao vistos em maior numero no quadrante craniolateral, seguido pelos quadrantes craniomedial, caudomedial e caudolateral;

considerando os rins direito e esquerdo, igual numero de ramos arteriais foi observado 9 vezes $(30,0 \%)$ e de ralzes venosas - 6 
Contribuição do estudo dos elementos vasculares, arteriais e venosos.do hilo renal

vezes $(20,0 \%)$, sendo que apenas 1 vez (3,3\%), ocorreu igual numero de ramos arteriais e ra!zes venosas à direito e à esquerda.

6)

os ramas arterieis encontram-se no rim direito, em posicgo predominantemence periferica - 16 vezes (53,3\%), exclusivamente periferica - 6 vezes $\left(20, C_{0}^{y}\right)$, predominantemente central - 6 vezes $(20,0 \%)$, igualmente central e periferica - 2 vezes $(S, 6 \%$ ) no rim esquerdo, predominantemente periferica - 16 vezes $(53,3 \%)$, exclusivamente periferica - 9 vezes $(30,0 \%)$, predominantemente central -2 vezes $(6,5 \%)$, exclisivamente cent:al - 1 vez (3,3\%) e igualmente central e periferica -2 vezes $(6,6 \%)$;

as ralzes venosas oeupam, no rim direito, posicao predominantemente periferica - 14 vezes $(46,6 \%)$, exclusivamente periférica - 9 vezes (30,0\%) predominantemente central 7 vezes $(23,3 \%)$, e no rim esquerdo, predominantemente periferica - 13 vezes $(43,3 \%)$, exclusivamente periferica - 10 vezes $\left(33,3^{\circ}\right)$, predominantemente central - 4 vezes $(13,3 \%)$ e igualmente central e periferica - 3 vezes $(3,3 \%)$;

a andise estatistica ngo revelou diferencas significantes, oo ntvel de 5,0\%, relativamente ao sexo, no que oiz respeito ao numero de ramos arteriais e raizes venosas, nem à direita nem à esquerda.

MARIANA, A.N.B. \& BORELLI, V. A contribution to the study of arterial and venous vascularization of the kidney nilus in zeou cattle (Nelore breeding). Rev. Fac. Med. Vet. Zootec. Univ. S. Paulo, $25(1): 53-70,1988$.

SUMMARY: This paper objetived to contribute to the knowledge of the arterial and venous elements in domestic animals kidneys. So, we have utilized 30 pairs of kidneys ootained from adult hnvines $15 \mathrm{males}$ and 915 females Nelore breed. After fixation in formol solution $(10 \%)$, the dissection are made to see the number anci pattern of the vascular branches. Concesning the right $k$ idney, the arterial branches numbered from 3 to 8 , with predominance of 4 ; and at the left one from 3 to 9 , with predominance of 4 about the vanous roots, in the right kidney, they numbered from $=$ to 10 , with predominance of 5 , and in the left kidney, from 3 to 10, with predominance of 6 . Generally, the arterial oranches and venous roots have showed at the hilus, peripherical situation. The statistical analysis didn't show diferences concerning the sexes.

UNITERMS: Anatomy of cattle; Kidney; Arteries; Veins 
QUADRO 1 - Ramos arteriais hilares to rim direito em bovinos da rapa Nelore. Săo Paulo, 1985.

QURDRRNTES

OBS CRL CRM CAL CAM

\section{LIMITE DLS QUADRANTES}

CPL-EPM ERL-CAL CAL-COM CPM-CAM

TOTAL

\begin{tabular}{|c|c|c|c|c|c|c|c|c|c|}
\hline 1 & - & 2 & - & 1 & - & - & - & - & 3 \\
\hline 2 & - & 2 & - & 1 & 1 & - & - & - & 4 \\
\hline 3 & - & 5 & - & 1 & - & - & - & - & 6 \\
\hline 4 & - & 2 & - & 1 & - & - & - & - & 3 \\
\hline 5 & - & 2 & 1 & 1 & - & - & - & - & 4 \\
\hline 6 & - & 3 & - & 1 & - & - & - & - & 4 \\
\hline 7 & - & 2 & - & 1 & - & - & - & - & 9 \\
\hline 8 & 1 & 4 & - & 1 & - & - & - & - & 7 \\
\hline 9 & - & 5 & - & 1 & - & - & - & - & 6 \\
\hline 10 & - & 3 & - & 1 & 1 & - & - & 1 & 6 \\
\hline 11 & 1 & 2 & - & 1 & - & - & - & - & 4 \\
\hline 12 & - & 5 & - & 1 & - & - & - & 1 & 7 \\
\hline 13 & - & 3 & - & 1 & - & - & - & - & 4 \\
\hline 14 & 1 & 5 & - & 1 & - & - & - & - & 7 \\
\hline 15 & - & 1 & - & 2 & - & - & - & - & 3 \\
\hline 16 & - & 2 & - & 2 & - & - & - & - & 4 \\
\hline 17 & 2 & 2 & - & 1 & 1 & - & - & - & 6 \\
\hline 18 & - & 3 & - & 1 & 1 & - & - & - & 5 \\
\hline 19 & 3 & 3 & - & 2 & - & - & - & - & $B$ \\
\hline 20 & - & 2 & - & 2 & - & - & - & - & 4 \\
\hline 21 & - & $\overline{1}$ & - & 2 & - & - & - & - & 3 \\
\hline 22 & 1 & 2 & - & 3 & - & - & - & - & 6 \\
\hline 23 & - & 5 & - & - & 1 & - & - & - & 5 \\
\hline 24 & - & 4 & - & - & - & - & - & - & 4 \\
\hline 25 & - & 4 & - & 1 & - & - & - & - & 5 \\
\hline 26 & - & 4 & 1 & - & - & - & - & - & 5 \\
\hline 27 & 1 & 2 & - & 1 & - & - & - & - & 4 \\
\hline 28 & - & 3 & 1 & 2 & - & - & - & - & 6 \\
\hline 29 & 1 & $\uparrow$ & - & 2 & - & - & - & - & 4 \\
\hline 30 & - & 2 & 1 & 1 & - & - & - & - & 4 \\
\hline $\begin{array}{l}\text { CRL } \\
\text { CRM } \\
\text { CRL } \\
\text { CAM }\end{array}$ & & $\begin{array}{l}\text { rante } \\
\text { rante } \\
\text { rante } \\
\text { rante }\end{array}$ & & $\begin{array}{l}\text { ion } \\
\text { ol } \\
\text { om }\end{array}$ & & & & & \\
\hline
\end{tabular}




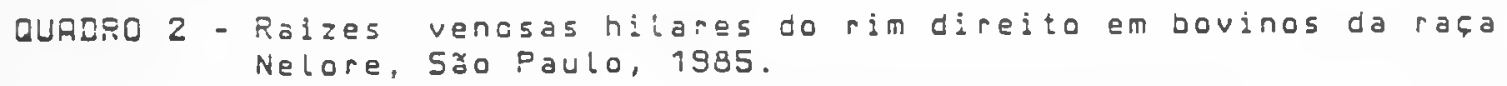

OUSADRANTES LIMITE DOS QUADRANTES
CRL CRM CAL CAM CRL-CRM CRL-LAL CAL-LAM CRM-CAM TOTRL

\begin{tabular}{|c|c|c|c|c|c|c|c|c|c|}
\hline 1 & 1 & 1 & 1 & 1 & $=$ & - & - & - & 4 \\
\hline 2 & 2 & - & 1 & 1 & 1 & - & 1 & - & 6 \\
\hline 3 & 2 & 3 & 1 & 2 & - & - & 1 & - & 9 \\
\hline 4 & 1 & 4 & 1 & 1 & 1 & - & - & - & $B$ \\
\hline 5 & - & 3 & - & 2 & - & - & - & - & 5 \\
\hline 6 & 3 & 1 & 2 & - & - & - & - & - & 6 \\
\hline 7 & 1 & - & 1 & 1 & 1 & - & - & - & 4 \\
\hline 8 & 1 & 3 & 1 & 1 & - & 1 & - & - & 7 \\
\hline 9 & 1 & 3 & 1 & 1 & 1 & - & 1 & - & 3 \\
\hline 10 & 1 & 2 & $\overline{-}$ & 1 & 1 & - & - & - & 5 \\
\hline 11 & 2 & 2 & 2 & 1 & - & - & - & - & 7 \\
\hline 12 & 2 & 3 & 3 & 1 & - & 1 & - & - & 10 \\
\hline 13 & 1 & 1 & 2 & 1 & - & - & - & - & 5 \\
\hline 14 & - & 1 & - & 1 & - & - & - & 1 & 3 \\
\hline 15 & - & 1 & - & 1 & - & - & - & - & 2 \\
\hline 16 & - & 2 & 1 & 1 & - & - & - & - & 4 \\
\hline 17 & 2 & 1 & 1 & 1 & - & $=$ & - & - & 5 \\
\hline 18 & - & 1 & - & 1 & 1 & - & - & - & 3 \\
\hline 19 & 2 & 2 & - & - & - & - & 1 & - & 5 \\
\hline 20 & 4 & 1 & 2 & 1 & - & 1 & - & - & 9 \\
\hline 21 & 2 & 1 & 1 & - & - & - & - & - & 4 \\
\hline 22 & 1 & 1 & 1 & 1 & - & - & 1 & - & 5 \\
\hline 23 & 2 & 2 & 1 & 1 & - & - & 1 & - & 7 \\
\hline 24 & 2 & 1 & 2 & 1 & - & - & - & - & 5 \\
\hline 25 & 2 & - & - & 1 & 1 & - & - & - & 4 \\
\hline 26 & - & 4 & 2 & 2 & - & 1 & - & - & 9 \\
\hline 27 & 1 & 1 & - & 1 & 1 & - & 1 & - & 5 \\
\hline 28 & 1 & 2 & 2 & 1 & - & - & - & - & 6 \\
\hline 29 & - & 1 & 1 & 2 & 1 & - & - & - & 5 \\
\hline 30 & 1 & 2 & 1 & 1 & - & - & - & - & 5 \\
\hline
\end{tabular}

CRL - quadrante craniolateral

CRM - quadrante craniomedial

CAL - quadrante caudolateral

CAM - quadrante caudomedial 
QUPDRO 3 - Ramos arteriais hilares do rim esquerdo em bovinos da rafa Nelore. Săo Paulo, 1985 .

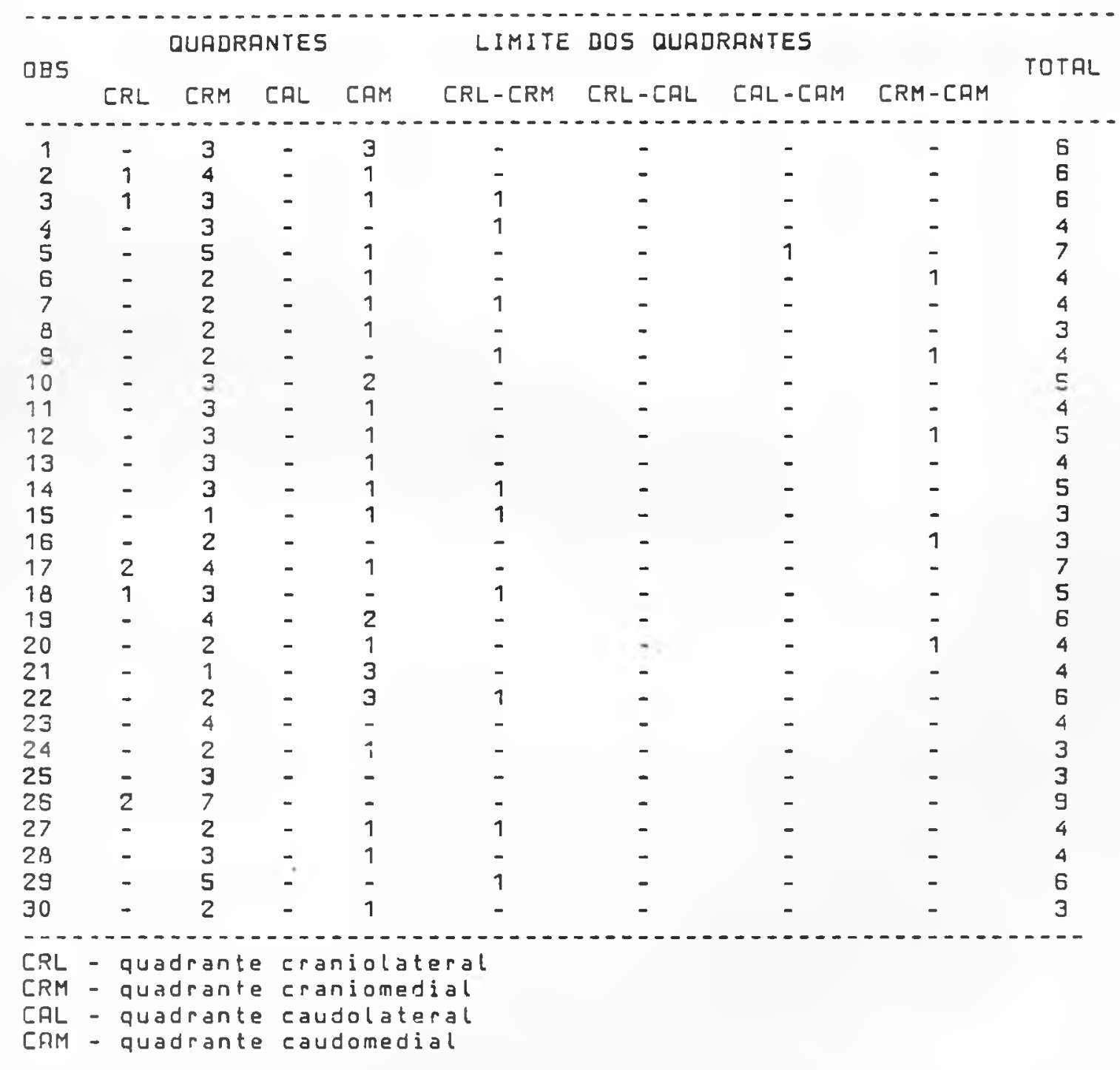

Rev. Fac. Meci. Vet. Zuutec. Liniv. 5. F̂aulo. 2j(1): j\$-10. 1988. 
60 MARIANA. A.N.B. \& BORELLI. V.

Contribuiçāo ao estudo dos elementos vasculares, arteriais e venosos.do hilo renal

QUADRo 4 - Ralzes venosas hilares do rim esquerdo em bovinos da raça Nelore. 5 a c Paubo, 1985.

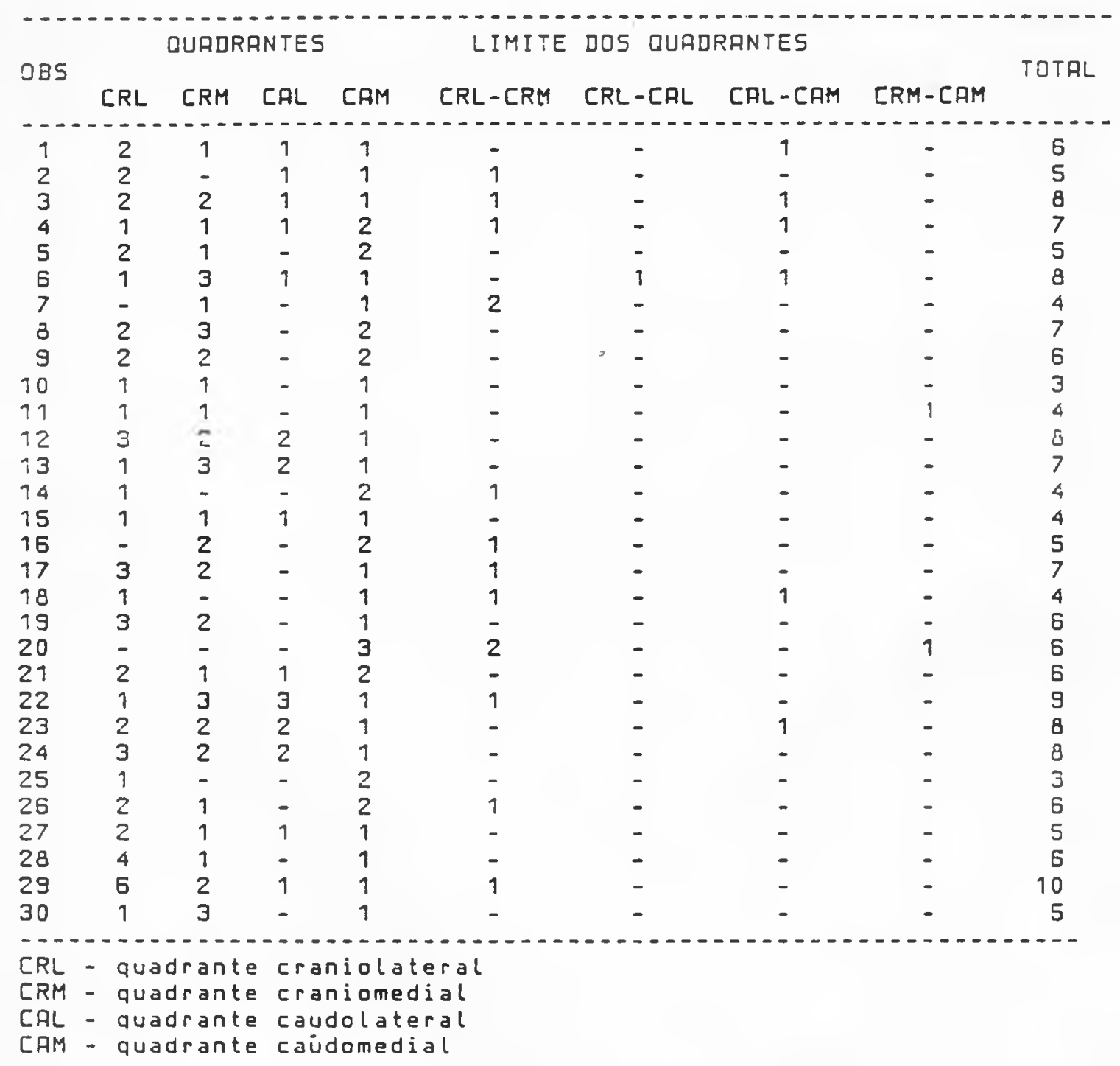


Figuras de 1 a 30 correspondentes as cjservacues de 1 a 30 ) - Representacres Esquemeticas dos ramos arteriais e das - jzes venosas das regiơs hilares, nos rins direito (D) e esaueioo (E) de bovinos da Faca Nelore, machos $(m)$ e fêneas (f), adultos, observados na face ventral ds orgå.

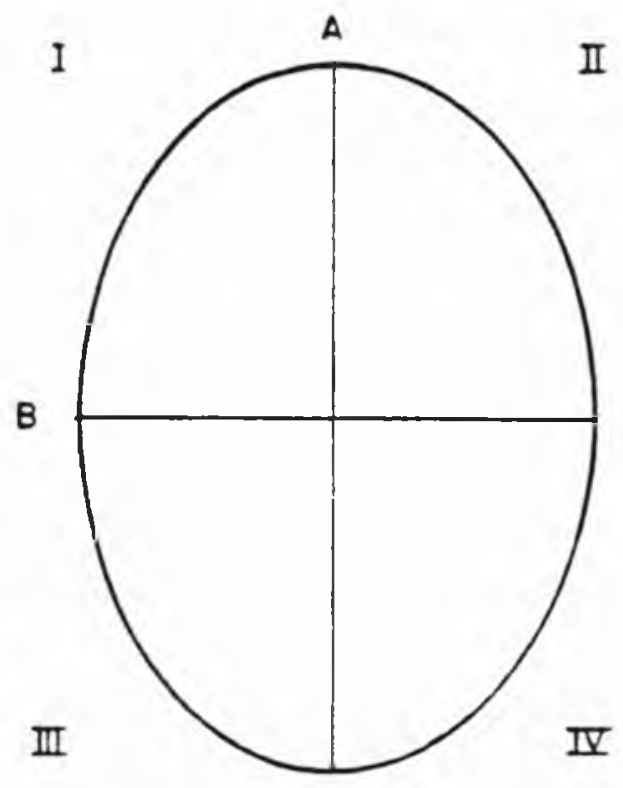

A. Lintia craniocaudal

B. Lintic laterolateral

I. Quadrante craniolateral

II . Quadrante craniomedial

III. Quadrante caudolateral

立. Quadrante caudomedial

$D$. Rim direito

$E$. Rim esquerdo

O. Ramo arterial

- Raiz venosa

$\triangle$ Ureter

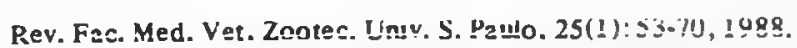



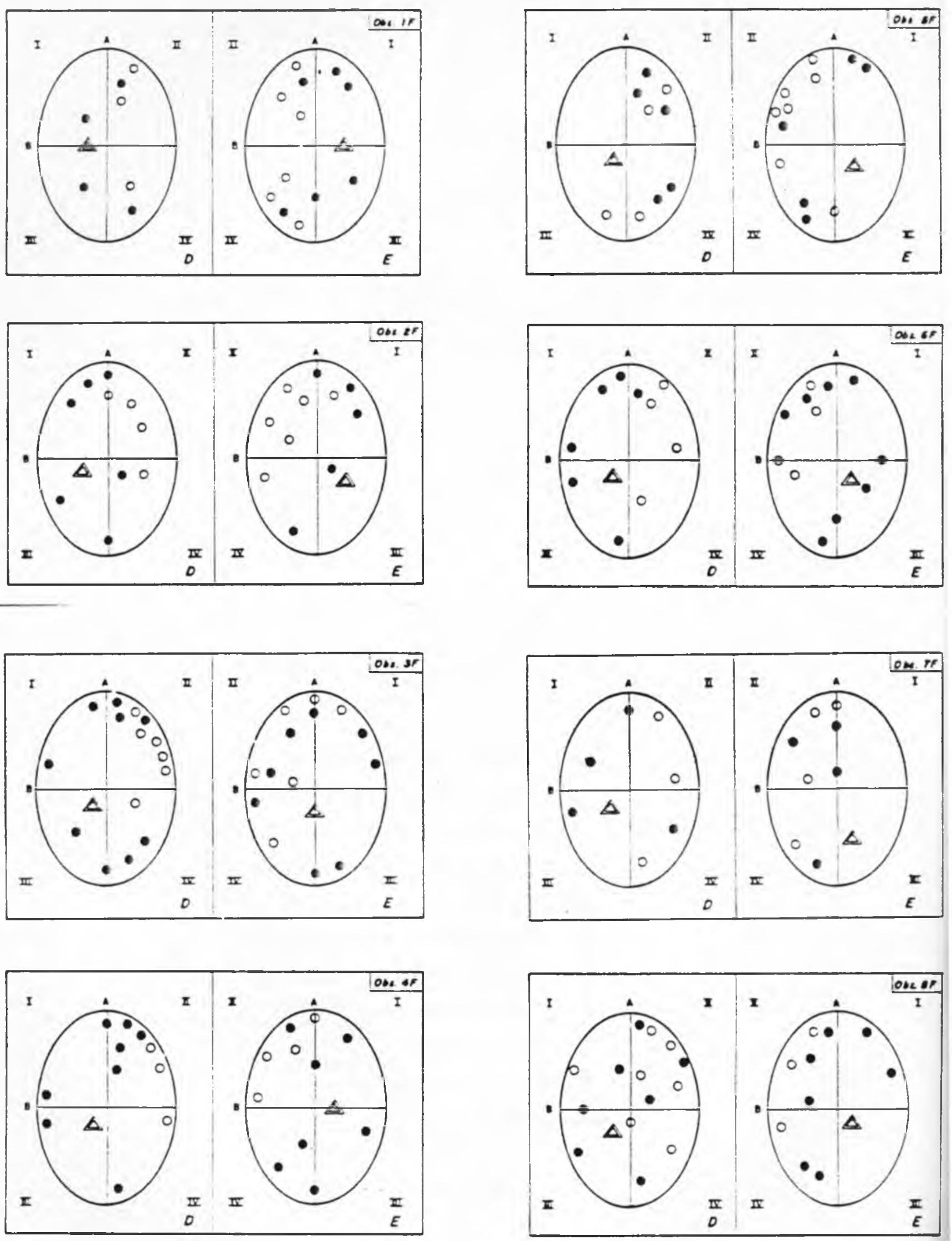

Rev. Far. Med. Vet Zeotec. Usir. S. Paudu. 25(1):53-70, 1988. 

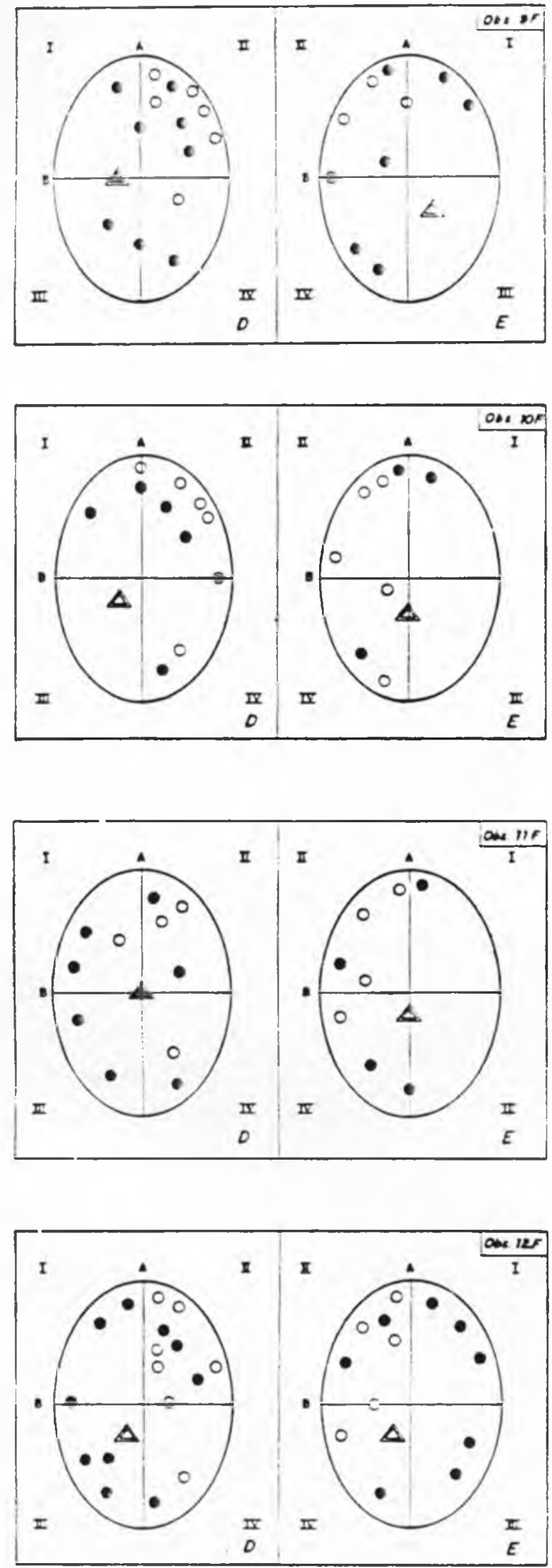
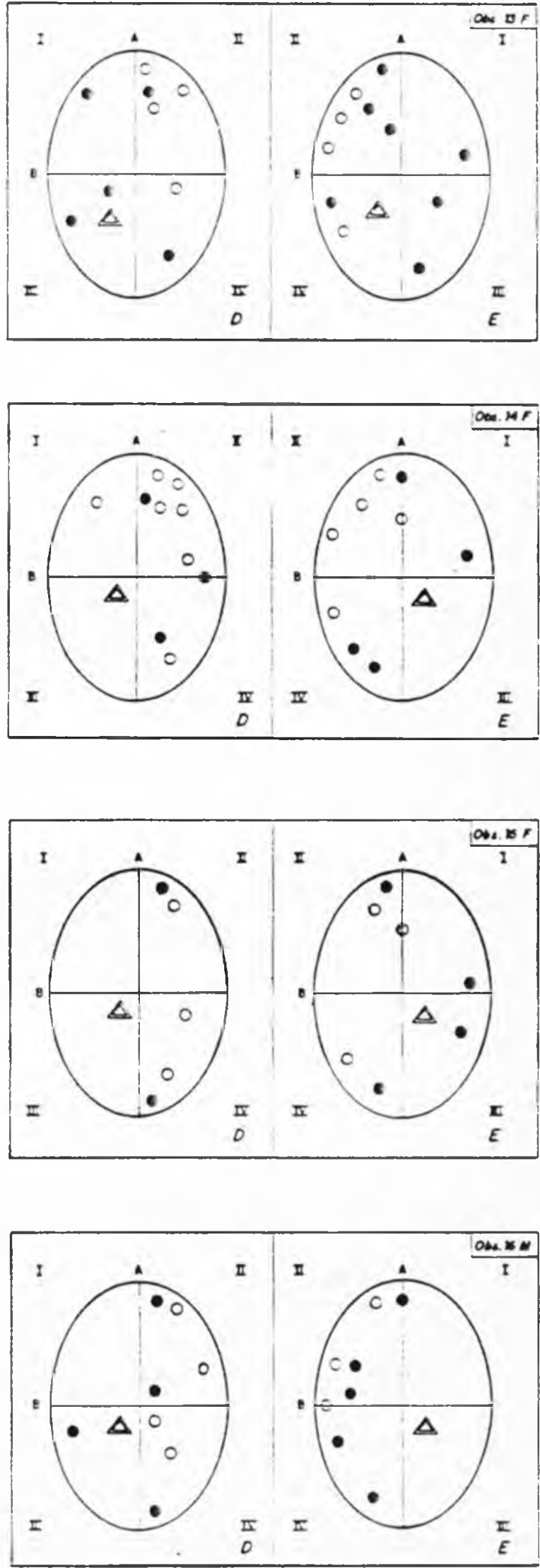

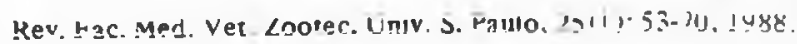


Contribuiçio ao estudo dos elementos vasculares, arteriais e venosos.do hilo renal
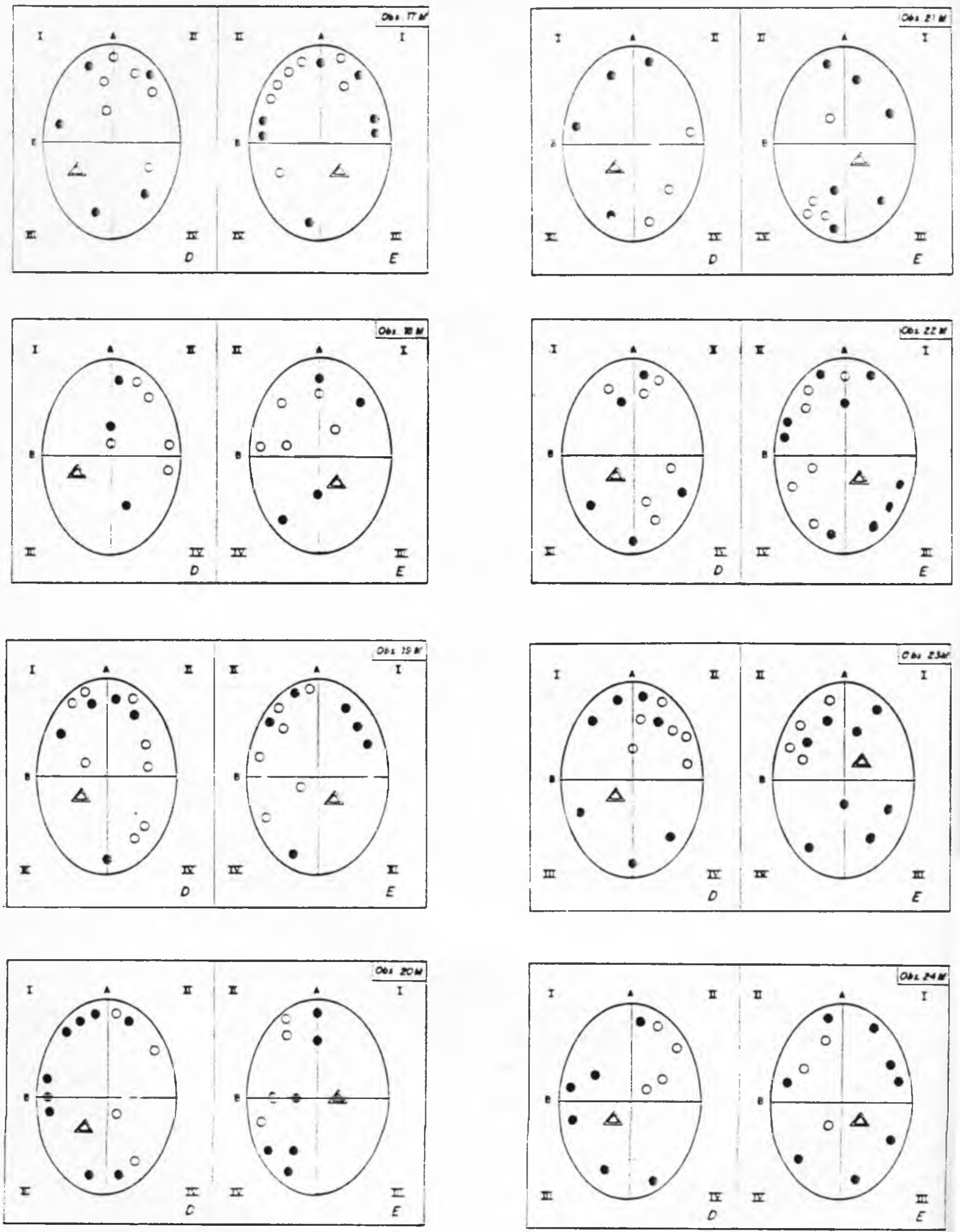

Rev. Fac. Med. Vet. Znnter, Inịy. S. Psulc. 25(1):53-70. 1988. 

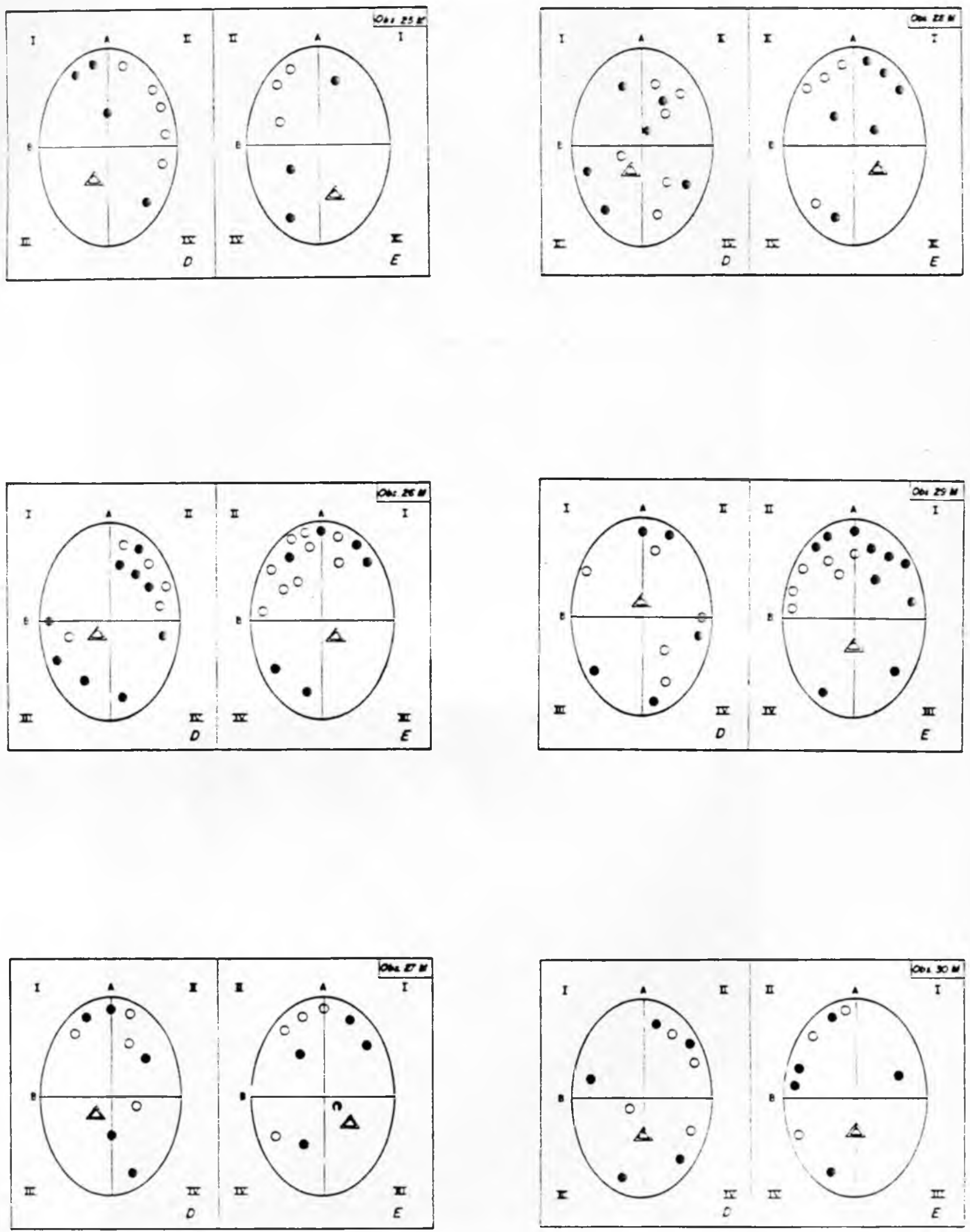

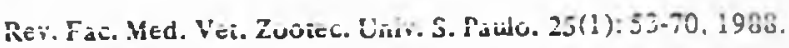




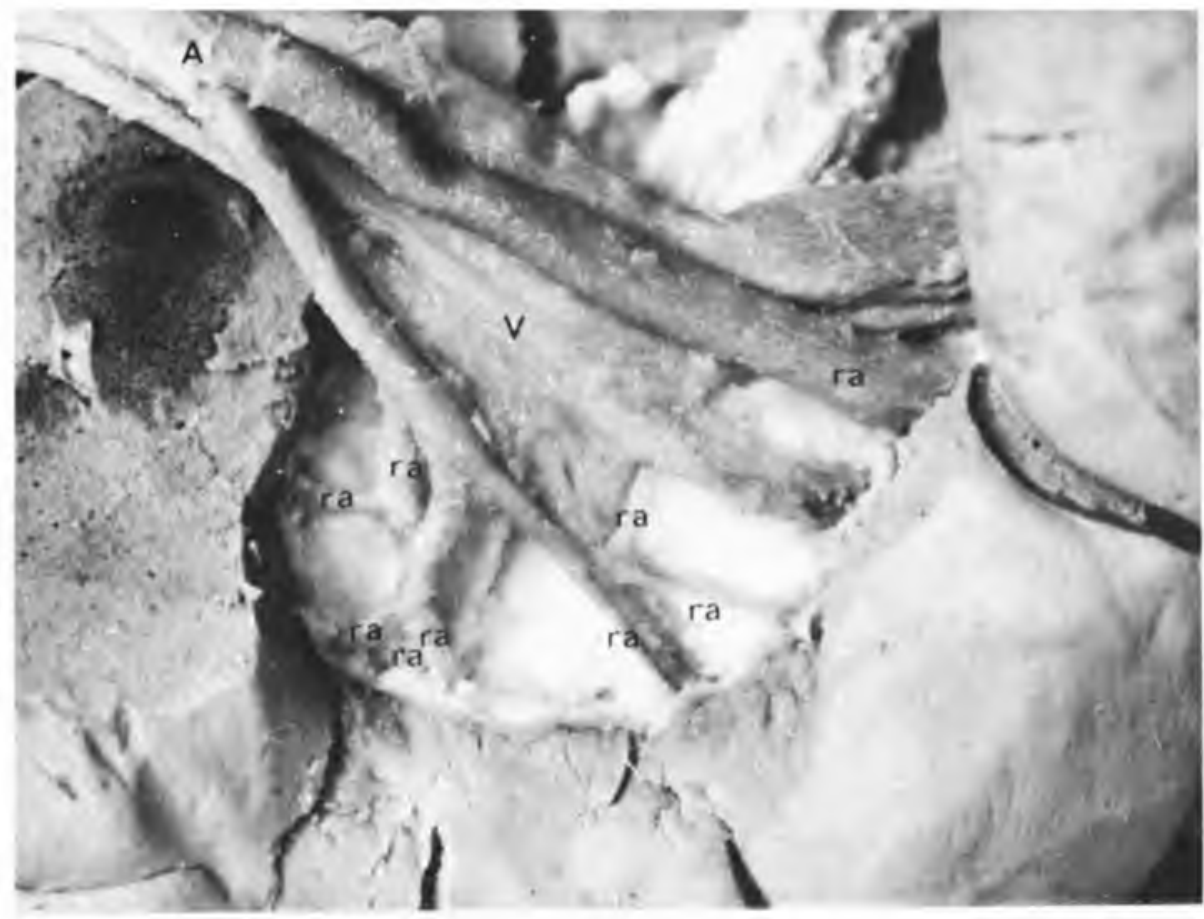

FIGURA 31 - Fotografia da face ventral da região hilar esquerda do rim de bovino, da raça Nelore (Obs. $26 \mathrm{~m}$ ) mostrando artéria renal (A) com nove ramos (ra) e veia renal (V) (Aumento $0,25 \mathrm{x}$ )

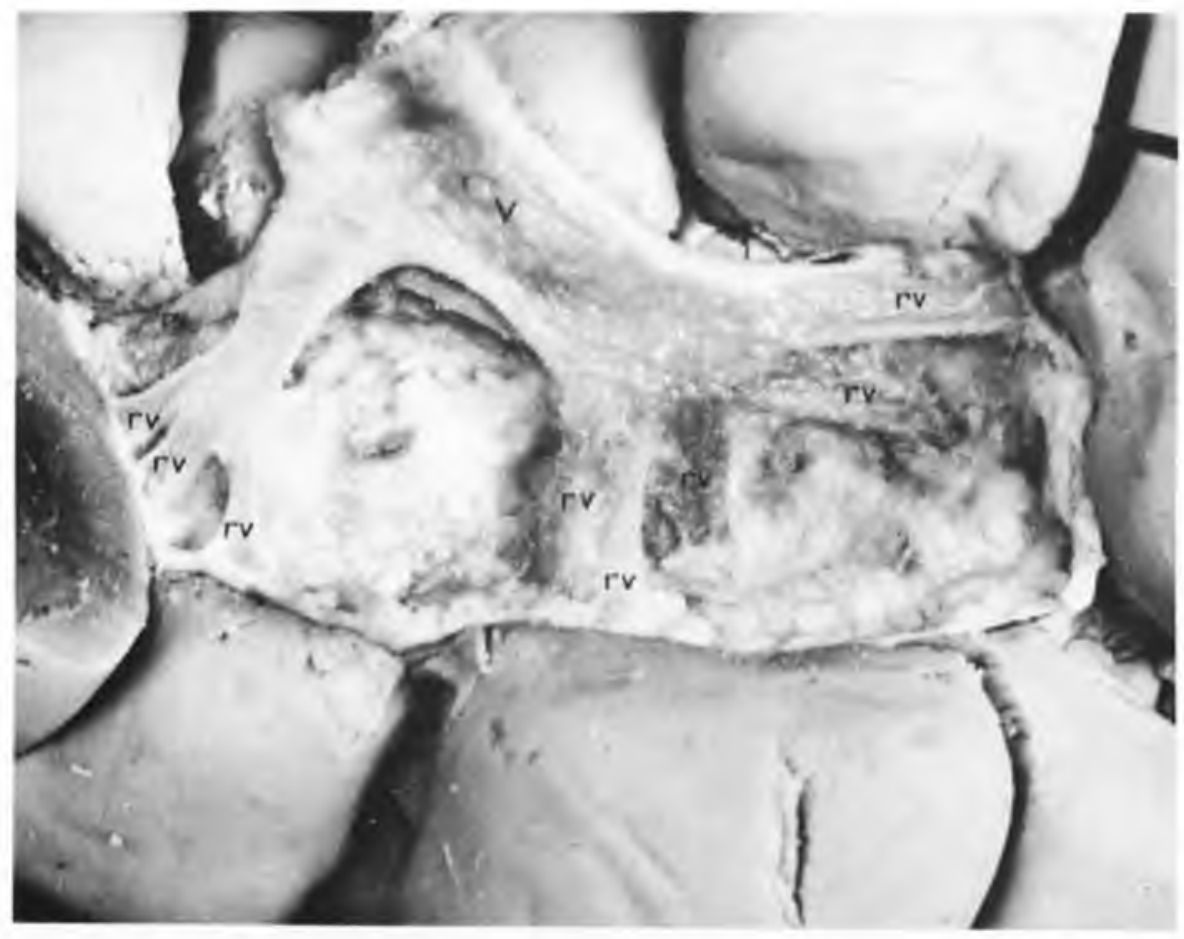

FIGURA 32 - Fotografia da face ventral da região hilar direita do rim de bovino, da raça Nelore (Obs. 20m) mostrando veia renal (V) com nove raízes (rv) (Aumento $0,25 \mathrm{x}$ ) 
1 - BOSJI, V.; CARADCNNA, G.B.; SPAMPANI, G.; UARALDI, L.; ZIMMERL, U. Trattato di anatomia veterinaria. Milano, Francesco Valiardi, s.d. v.2, D.207-340.

2 - BRUNI, A. C. \& ZIMMERL, U. Anatomia degli animali domestici. Mijlano, Francesco Vallardi, 1947. v. 2, p. $153-154$.

3 - DOBBERSTEIN, J. \& HOFFMANN, G. Lehrouch der verglejchenden Anatomie der Haustiere. Leipzig, 5. Hirzel, 1964. v. 3, p.50,70.

4 - EllenBerger, $W$. \& BAUM, $H$. Handbuch der vergleicheden Anatomie der Haustiere. Berlin, Julius 5pringer, 1992. p.696731 .

5 - FAVILLI, N. Nozione comparate di anatomia e fisiologia degli animali rurali. Torino, Unione Tipografica - Editrice Torinese, 1931. p. 449 .

6 - GETTY, R. Sisson and Grossman's the anatomy of the domestic animals. 5.ed. Philadelphia, W. 3. Saunders, 1975. p.985.

7 - GOHZFLEZ Y GARCIA, J. \& GONZALEZ fLVAREZ, R. Anatomia comparada de los animales domesticos. 7.ed. Madrid, Grafica Canales, 1967. p.548.

8 - KOCH, T. Lehrbuch der VeterinarAnatomie. Jena, Gustav Fischer, 1965. v.3, p.123.

9 - LEISERING, A.G.T. \& MUELLER, ᄃ. E.F. Gurlt's Handbuch der vergleichenon Anatomie der Haus-5auget iere. 5.Auf. Berin, August Hirschwald, 1873 . p.620, 670.
10 - Lepoutre, L. Notes du cours d'anatomie comparee des animaux domestiques. Gemolelix, d. Dueulot, 1ミ21. p.164.

11 - Lesgre, $F . X$. Precis d'anatomie comparie des aninaux domestiques. Paris, J.E. Baillière, 1923. v.2, p.78-80.

12 - MARTIN, P. Lehrbuch der Anatomie der Haustiere. Stuttgard, Schickhardt \& Ebner, 1904. v.2, ค.861, 910 .

13 - MARTIN, P. \& SCHAUDER, W. Lehrbuch der Anatomie der Haustlere. Stuttgart, Schicktiard 8 Ebner, 1938. v. 3, t.3, p.277-278.

i4 - MASS:I, K. Anztomia compziada das animeis domestises. ioed. Tokio, Yokenoo, 1550. v. 1 , p. 189 .

15 - MONGIRRDINO, T. Trattato di anatomia topografica dej mammiferl domestici. Torino, Luigi Delgrosso, 1903. p.192.

TE - montane, l. \& sourjelle, E. Ana:omie régionale des animaux domestiques. Paris, J.B. Zaillière, 1917. v.2, p.298-300.

17 - NICKEL, R.; SCHUMMER, A .; SEIFERLE, E.; SACK, W.O. The viseera of the domestic mammals. Gerlin, Paul Parey, 1973. p.287.

18 - SCHWARZE, E. 3 5CHRODER, L. COMpendio de anatomia vetepincria. Zaragoza, Acribia, 1972. v.3, p. $72-98$.

19 - SISSON, 5. \& GROSSMAN, J.D. Anatomia de los animales domesticos. Kev. Fac. Wied. Vet. Zontec. Üniv. S. Yaulo, 25(1):53-70. 1988. 
70 MARIANA. A.N.B. \& BORELLI.V.

Contribuição a estudo dos elementos vasculares. artcriais e venosos.do hilo renal
4.ed. Barcelona, Salvat, 1953.
e Zooteenia da USPJ ว. 558 .

20 - TEIXEIRA FILHO, A. Ccntribuitao ao estudo dos elementos vasculares, arteriais e venosos, do hilo renal, em bovinas da paea

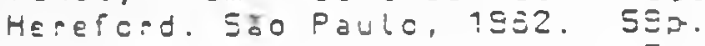
[Dissertacao de mestrado - Faculdade de Medicina Veterinaria
21 - ZIMMERL, U.; BRUNI, R.C.; CRRGDON- NA, E.B.; MANNU, P.; PREZILZO, L. Trattato di anatomia veteri- naria. Milano, Francesco Val- lardi, 1930. v. 3, p.15-18.

Recebido para publicac9o em $28 / 05 / 87$ Aprovado para publicacao em $02 / 11 / 87$ 